\title{
Leftist Canonical Ordering
}

\author{
Melanie Badent ${ }^{1}$, Michael Baur ${ }^{2}$, Ulrik Brandes ${ }^{1}$, and Sabine Cornelsen ${ }^{1}$ \\ 1 Department of Computer \& Information Science, University of Konstanz \\ \{Melanie.Badent,Ulrik.Brandes, Sabine.Cornelsen\}@uni-konstanz.de \\ 2 Department of Computer Science, Universität Karlsruhe (TH) \\ baur@informatik.uni-karlsruhe.de
}

\begin{abstract}
Canonical ordering is an important tool in planar graph drawing and other applications. Although a linear-time algorithm to determine canonical orderings has been known for a while, it is rather complicated to understand and implement, and the output is not uniquely determined. We present a new approach that is simpler and more intuitive, and that computes a newly defined leftist canonical ordering of a triconnected graph which is a uniquely determined leftmost canonical ordering.
\end{abstract}

\section{Introduction}

Canonical vertex orderings were introduced by de Fraysseix, Pach, and Pollack 13/14 and are the backbone of several algorithms for planar graphs, including graph drawing algorithms 2 2|3|4|8|9|10|16|17|18|19|20|22|23|28|27|29|30|, graph encoding [111/26], construction of realizers, spanners, or orderly spanning trees [5]6|7|15|31|32|33, and more [12|25|34.

Kant [28 generalized canonical orderings to triconnected graphs. While several implementations of the linear-time algorithm of Kant are available, this algorithm is neither trivial to code, nor is its correctness easily understood. Based on a simple and intuitive criterion, we present a new algorithm that might further broaden the scope of adoption and ease teaching.

Since a triconnected graph can have many canonical orderings, we introduce the leftist (and rightist) canonical ordering that is uniquely determined. The leftist canonical ordering is in particular a leftmost canonical ordering.

The main advantage of our algorithm compared to the algorithm in 28 is that we do not use the dual graph nor any face labels. Further, we compute the unique leftist canonical ordering from scratch, i. e., without any reordering, and we compute it from the low numbers to the high numbers contrary to the previous algorithm that builds the canonical ordering from the end by shelling off paths from the outer face. A similar approach for biconnected canonical orderings can be found in [24]. We also give a detailed pseudocode such that it can be easily implemented. Finally, our proof of correctness includes a new proof of the existence of a canonical ordering for triconnected graphs.

The paper is organized as follows. Canonical orderings are defined in Sect. 2 The new algorithm and its linear-time implementation are described in Sects. 3 and 4, respectively.

D. Eppstein and E.R. Gansner (Eds.): GD 2009, LNCS 5849, pp. 159-170 2010.

(C) Springer-Verlag Berlin Heidelberg 2010 


\section{Preliminaries}

Throughout this paper, let $G=(V, E)$ be a simple undirected graph with $n$ vertices, $n \geq 3$, and $m$ edges. We assume that $G$ is planar and triconnected, hence it has a unique planar embedding up to the choice of the outer face. For a subset $V^{\prime} \subseteq V$ we denote by $G\left[V^{\prime}\right]$ the subgraph of $G$ induced by $V^{\prime}$. By $\operatorname{deg}_{G}(v)$ we denote the number of edges of $G$ that contain $v$. A path is a sequence $P=\left\langle v_{0}, \ldots, v_{\ell}\right\rangle$ of distinct adjacent vertices, i. e., $\left\{v_{i}, v_{i+1}\right\} \in E$. We also denote the set $\left\{v_{0}, \ldots, v_{\ell}\right\}$ by $P$.

Canonical orderings were introduced originally for triangulated graphs by de Fraysseix et al. 1314. The following rephrases Kant's generalization to triconnected graphs [28].

Definition 1 (canonical ordering). Let $\Pi=\left(P_{0}, \ldots, P_{r}\right)$ be a partition of $V$ into paths and let $P_{0}=\left\langle v_{1}, v_{2}\right\rangle, P_{r}=\left\langle v_{n}\right\rangle$ such that $v_{2}, v_{1}, v_{n}$ is a path on the outer face of $G$ in clockwise direction. For $k=0, \ldots, r$ let $G_{k}=G\left[V_{k}\right]=\left(V_{k}, E_{k}\right)$ be the subgraph induced by $V_{k}=P_{0} \cup \cdots \cup P_{k}$, let $C_{k}$ be the outer face of $G_{k}$. Partition $\Pi$ is a canonical ordering of $\left(G, v_{1}\right)$ if for each $k=1, \ldots, r-1$ :

1. $C_{k}$ is a simple cycle.

2. Each vertex $z_{i}$ in $P_{k}$ has a neighbor in $V \backslash V_{k}$.

3. $\left|P_{k}\right|=1$ or $\operatorname{deg}_{G_{k}}\left(z_{i}\right)=2$ for each vertex $z_{i}$ in $P_{k}$.

$P_{k}$ is called a singleton if $\left|P_{k}\right|=1$ and a chain otherwise.

A canonical ordering $\Pi$ is refined to a canonical vertex ordering $v_{1}, \ldots, v_{n}$ by ordering the vertices in each $P_{k}, k>0$, according to their clockwise appearance on $C_{k}$ (see Figs. 1(a) 1(c).

The following observations help build an intuitive understanding of canonical orderings. Note that Propositions 4 and 5 of Lemma 1 are part of Kant's original definition.

Lemma 1. For $k=1, \ldots, r-1$ :

1. $P_{k}$ has no chord.

2. For each vertex $v$ in $P_{k}$ there is a $v$ - $v_{n}$-path $v=v_{k_{0}}, \ldots, v_{k_{\ell}}=v_{n}$ where each $v_{k_{i}}$ is in $P_{k_{i}}$ and $k_{i}<k_{j}$ for $0 \leq i<j \leq \ell$. Especially:

(a) $G\left[V \backslash V_{k}\right]$ is connected.

(b) If $\operatorname{deg}_{G_{k}}(v)=2$, then $v$ is in $C_{k}$.

(c) $P_{k}$ is on $C_{k}$.

3. (a) A singleton $P_{k+1}$ and a path of $C_{k}$ bound some faces or

(b) a chain $P_{k+1}$ and a path of $C_{k}$ bound one face.

4. $G_{k}$ is biconnected.

5. If $v, w$ is a separation pair of $G_{k}$, then both are on $C_{k}$.

Proof. The properties are directly implied by the fact that $G$ is triconnected and by the definition of a canonical ordering.

Remark 1. Two incident faces of a triconnected planar graph share one vertex or one edge. Especially, no face has a chord. 


\subsection{Leftmost Canonical Ordering}

Kant 28 introduced a leftmost and rightmost canonical ordering of $G$. Let $P_{0}, \ldots, P_{k}$ be a sequence of paths that can be extended to a canonical ordering of $G$. A path $P$ of $G$ is a feasible candidate for the step $k+1$ if also $P_{0}, \ldots, P_{k}, P$ can be extended to a canonical ordering. Let $v_{1}=c_{1}, c_{2}, \ldots, c_{q}=v_{2}$ be the vertices from left to right on $C_{k}$. Let $c_{\ell}$ be the neighbor of $P$ on $C_{k}$ such that $\ell$ is as small as possible. We call $c_{\ell}$ the left neighbor of $P$.

Definition 2 (leftmost canonical ordering). A canonical ordering $P_{0}, \ldots, P_{r}$ is called leftmost (rightmost) if for $k=0, \ldots, r-1$ the following is true. Let $c_{\ell}$ be the left neighbor of $P_{k+1}$ and let $P_{k^{\prime}}, k+1 \leq k^{\prime} \leq r$, be a feasible candidate for the step $k+1$ with left neighbor $c_{\ell^{\prime}}$. Then either (1) $\ell \leq \ell^{\prime}\left(\ell \geq \ell^{\prime}\right)$ or (2) there is an edge between $P_{k+1}$ and $P_{k^{\prime}}$ (see Fig. 1(b)).

Note that once a canonical ordering is known a simple linear-time algorithm can be used to rearrange its paths so that it becomes leftmost [28]. Also note that Kant did not use Condition 2 of a leftmost canonical ordering in his definition, however, he used it in his reordering algorithm. While leftmost canonical orderings are particularly useful for many applications, we stress that the rearrangement is applicable to any canonical ordering and that a leftmost canonical ordering is only unique with respect to a given partiton.

\subsection{Leftist Canonical Ordering}

In the leftist canonical ordering we add in each step the leftmost possible path where the choice is not only within an already given partition.

Definition 3 (leftist canonical ordering). A canonical ordering $P_{0}, \ldots, P_{r}$ is called leftist (rightist) if for $k=0, \ldots, r-1$ the following is true. Let $c_{\ell}$ be the left neighbor of $P_{k+1}$ and let $P$ be a feasible candidate for the step $k+1$ with left neighbor $c_{\ell^{\prime}}$. Then $\ell \leq \ell^{\prime}\left(\ell \geq \ell^{\prime}\right)$ (see Figs. 1(c) and 1(a)) .

Note that a feasible candidate for the step $k+1$ needs not to be a feasible candidate for the step $k+2$ anymore. Also note that the leftist canonical ordering is unique irrespective of a given partition and it is a leftmost canonical ordering. A leftist canonical ordering can also be found by choosing always the rightmost face or singleton in the algorithm of Kant [28]. A similar concept related to Schnyder realizers without clockwise cycles was defined for triangulated graphs in [6].

\section{New Algorithm}

Starting from $P_{0}=\left\langle v_{1}, v_{2}\right\rangle$, we build the canonical ordering by adding $P_{1}, \ldots, P_{r}$ in this order. In step $k+1$, the "belt" around $G_{k}$, i. e., the sequence of vertices not in $G_{k}$ that lie on faces incident to $G_{k}$ is considered. Then, a candidate not causing any "self-intersection" within the belt is chosen. Before we give the details, we start with a recursive definition of which paths will be considered in the step $k+1$. 


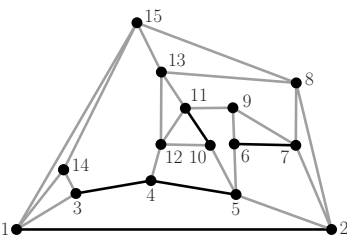

(a)

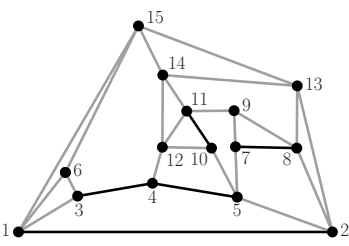

(b)

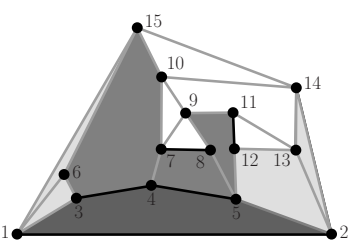

(c)

\section{CANDidate.Chain \\ $\downarrow$ \\ BELT $\frac{(1,15)(15,6)(6,1)(1,6)(6,3)(3,1)}{(193}$ \\ $(2,5)(5,12)(12,13)(13,2)|(2,13)(13,14)(14,2)|(2,14)(14,15)(15,1)$}

EXTENSION

Fig. 1. Different canonical orderings (black paths are chains). (a) Rightist canonical ordering. (b) Leftmost canonical ordering respecting the ordering in (a). (c) Leftist canonical ordering and its construction. The light and dark grey faces are the belt of $G_{0}$. The next candidate in Algorithm 3 is $P_{1}=\langle 3,4,5\rangle$. Algorithm [5 substitutes the dark grey face by the middle grey faces, i. e., by the ExTEnsion found by Algorithm 4

Definition 4 (cut faces and locally feasible candidates). $P_{0}=\left\langle v_{1}, v_{2}\right\rangle$ is a locally feasible candidate. Let $P_{0}, \ldots, P_{k}$ be a sequence of locally feasible candidates and $V_{k}, G_{k}$, and $C_{k}$ as in Definition 1 . $A$ cut face $f$ of $G_{k}$ is an inner face of $G$ that is incident to some vertex on $C_{k}$ but is not a face of $G_{k}$. Let $P_{f}$ be the clockwise sequence of vertices incident to $f$ that are not in $V_{k}$. If $f$ is incident to an edge on $C_{k}$, then $f$ is called a candidate face and $P_{f}$ is called a candidate for the step $k+1$. A candidate face $f$ and the candidate $P_{f}$ are locally feasible for the step $k+1$ if

1. $v_{n}$ is not in $P_{f}$ or $P_{0}, \ldots, P_{k}, P_{f}$ is a partition of $V$,

2. $G\left[V \backslash\left(V_{k} \cup P_{f}\right)\right]$ is connected, and

3. $P_{f}$ is a singleton or the degree of each vertex of $P_{f}$ in $G\left[V_{k} \cup P_{f}\right]$ is two.

In the remainder of this section, we will see that the locally feasible candidates are exactly the feasible candidates. We start with the following lemma which is a direct consequence of Definitions 1 and 4 and the triconnectivity of $G$.

\section{Lemma 2}

1. A canonical ordering is a sequence of locally feasible candidates.

2. If a sequence of locally feasible candidates partitions the whole vertex set of a triconnected graph, it is a canonical ordering.

In what follows, we consider the vertices on $C_{k}$ to be from left to right between $v_{1}$ and $v_{2}$. Accordingly, we also consider the cut faces from left to right: A cut edge of $G_{k}$ is an edge of $G$ that is incident to one vertex in $V_{k}$ and one vertex in $V \backslash V_{k}$. Let $f$ and $f^{\prime}$ be two cut faces. Let $c$ and $c^{\prime}$, respectively, be the leftmost vertices on $C_{k}$ that are incident to $f$ and $f^{\prime}$, respectively. We say that $f$ is to the left of $f^{\prime}$ if $c$ is to the left of $c^{\prime}$ on $C_{k}$ or if $c=c^{\prime}$, then the cut edges of $f$ are to the left of the cut edges of $f^{\prime}$ in the incidence list of $c$. 


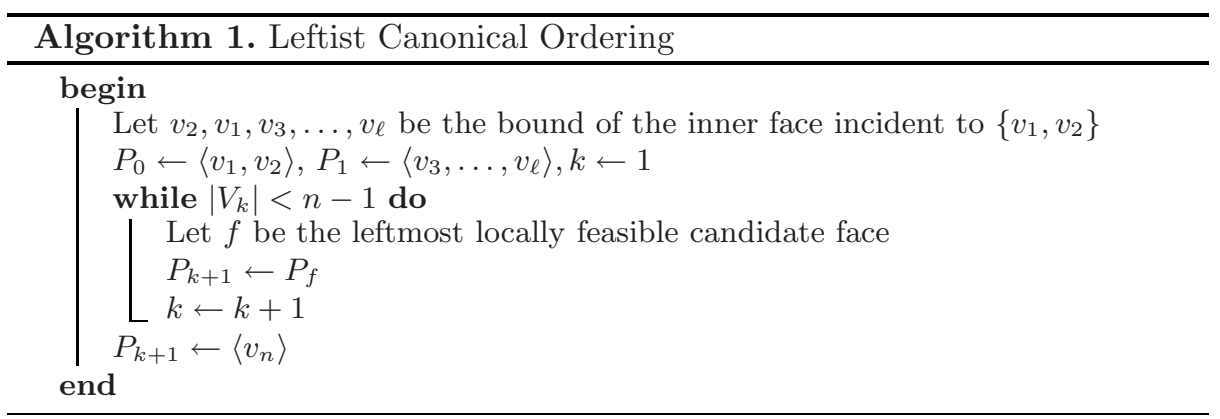

Corollary 1. If Algorithm 1 terminates, it computes the leftist canonical ordering of a triconnected planar graph.

Before we prove that in each step there exists a locally feasible candidate face, we describe locally feasible candidates in terms of "self-intersection" of the belt. Let $P_{0}, \ldots, P_{k}$ be a sequence of locally feasible candidates. The belt of $G_{k}$ is the sequence of vertices not in $G_{k}$ that are incident to the cut faces of $G_{k}$ from left to right. I. e., let $f_{1}, \ldots, f_{s}$ be the cut faces of $G_{k}$ ordered from left to right. Let $P_{f_{0}}$ be the vertices in $V \backslash V_{k}$ that are incident to the outer face in counterclockwise order. Then, the concatenation of $P_{f_{1}}, \ldots, P_{f_{s}}$ and $P_{f_{0}}$ is the belt of $G_{k}$. Consider Fig. $1(\mathrm{a})$. Then, $P_{2}=\langle 6,7\rangle, P_{3}=\langle 8\rangle$, and the belt of $G_{3}$ is $15,14|14| 14,15,13,12|12,10| 10,11,9|9| 9,11,13|13,15| 15$.

Definition 5 (forbidden, singular, stopper). A vertex $v$ of the belt of $G_{k}$ is

- forbidden if $v$ does not occur consecutively in the belt of $G_{k}$,

- singular if $v$ occurs more than twice in the belt of $G_{k}$ and its occurrence is consecutive, and

- a stopper if it is forbidden or singular.

In the above example, 15, 13, and 11 are forbidden and 14 and 9 are singular vertices. Note that $v_{n}$ is always the first and last vertex of the belt. Hence, it remains forbidden until the end. It will turn out that the locally feasible candidates are those that do not contain a stopper or that are singular singletons.

Lemma 3. Let $P_{0}, \ldots, P_{k}$ be a sequence of locally feasible candidates. Let $f$ be a candidate face for the step $k+1$ and let $P=P_{f}$.

1. If a vertex $v$ of $P$ is adjacent to more than two vertices in $V_{k} \cup P$, then $v$ occurs more than twice in the belt.

2. If $G\left[V \backslash\left(V_{k} \cup P\right)\right]$ is not connected, then $P$ contains a forbidden vertex.

3. If a vertex $v$ of $P$ is singular, then $v$ is a locally feasible singleton.

4. If $P$ contains a forbidden vertex $v$, then $G\left[V \backslash\left(V_{k} \cup P\right)\right]$ is not connected or $P$ contains another vertex with more than two neighbors in $V_{k} \cup P$.

Proof. 1. Let $e$ be an edge incident to $v$ and a vertex in $V_{k} \cup P$ that is not incident to $f$. By Remark 1, edge $e$ is a cut edge and hence incident to two cut faces. Thus, $v$ is incident to at least three cut faces. 
2. Let $W$ be the set of vertices in a connected component of the graph induced by $V \backslash\left(V_{k} \cup P\right)$ and not containing $v_{n}$. Since $V \backslash V_{k}$ was connected, $W$ is adjacent to $P$ and there is a path from $P$ to $v_{n}$ not intersecting $W$. By the triconnectivity of $G$, there is an edge between $W$ and the part of $C_{k}$ not contained in $f$. Further, there is at least a third vertex on $C_{k} \cup P$ adjacent to $W$. Let $w$ be the rightmost vertex on $C_{k} \cup P$ that is adjacent to $W$ and let $v$ be the leftmost such vertex. Assume that $w$ is on $C_{k}$. Then $v$ is on $P$. Consider the face $f^{\prime}$ containing $v$ and $w$. Then, the belt contains some vertices of $W$ between the occurrences of $v$ for the belt faces $f$ and $f^{\prime}$ (see Fig. 2(a).

3. If $v$ is singular, then it is a candidate. By Proposition $2, G\left[V \backslash\left(V_{k} \cup\{v\}\right)\right]$ is connected.

4. Since $v$ is forbidden, there is a cut face $f^{\prime}$ containing $v$ and a cut face $h$ between $f$ and $f^{\prime}$ such that $P_{h}$ contains a vertex $w \neq v$. If $w$ is not incident to $f$, then $w$ and $v_{n}$ are in two connected components of $G\left[V \backslash\left(V_{k} \cup P\right)\right]$ (see Fig. 2(b) . So assume now that for all faces $h^{\prime}$ between $f$ and $f^{\prime}$ the path $P_{h^{\prime}}$ contains only vertices incident to $f$. Among these faces let $h$ be the face that is next to $f$. By Remark 1, $P_{h}$ consists of one vertex $w \neq v$ and $w$ is singular (see Fig. 2(c)).

Corollary 2. 1. A candidate that is a chain is locally feasible if and only if it does not contain any stopper.

2. A vertex of the belt is a locally feasible singleton if and only if it is singular.

For example, the locally feasible candidates for the step $k+1=4$ in Fig. 1(a) are $\langle 14\rangle,\langle 12,10\rangle$, and $\langle 9\rangle$.

Theorem 1. Algorithm 1 computes the leftist canonical ordering of a triconnected planar graph.

Proof. By Lemma 1] it remains to show that in each step of the algorithm there is a locally feasible candidate. By Corollary 22, if there are any singular vertices, we have a locally feasible candidate. So, assume now we do not have any singular vertices. By Corollary 2 we have to show that there is a candidate that does not contain any forbidden vertex.

Let $f$ be a candidate face and let $P=P_{f}$. Assume that $P$ contains a forbidden vertex $v$. Let $f^{\prime}$ be a cut face containing $v$ such that the belt contains a vertex other than $v$ between the occurrence of $v$ in $P_{f}$ and the occurrence of $v$ in $P_{f^{\prime}}$. Let $f, h_{1}, \ldots, h_{\alpha}, f^{\prime}$ be the sequence of cut faces between $f$ and $f^{\prime}$. We show by induction on the number of forbidden vertices in $P_{h_{1}}, \ldots, P_{h_{\alpha}}$ that there is a locally feasible candidate among $P_{h_{1}}, \ldots, P_{h_{\alpha}}$.

By the choice of $f^{\prime}$ and by triconnectivity of $G$, there is at least one $i=1, \ldots, \alpha$ such that $P_{h_{i}}$ is a candidate that does not contain $v$. If $v$ is the only forbidden vertex in $P_{h_{1}}, \ldots, P_{h_{\alpha}}$, then $P_{h_{i}}$ is locally feasible.

If $P_{h_{i}}$ contains a forbidden vertex $w$ (recall that by our assumption there are no singular vertices), there is a cut face $h \neq h_{i}$ among $f, h_{1}, \ldots, h_{\alpha}, f^{\prime}$ incident to $w$ such that the belt contains a vertex other than $w$ between the occurrence 


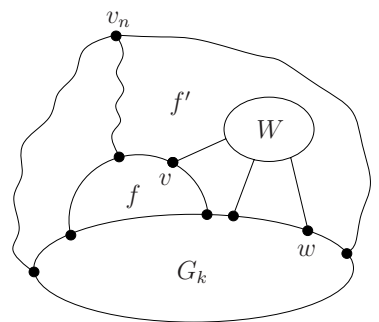

(a)

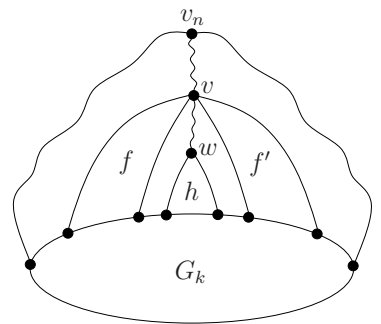

(b)

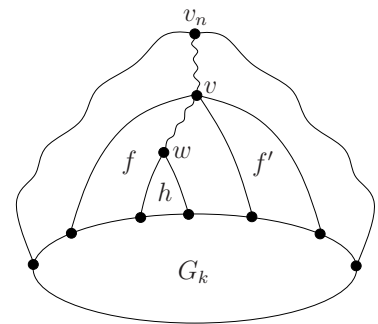

(c)

Fig. 2. Illustration of the proof of Lemma 3 (a) $W$ is a connected component of $G\left[V \backslash\left(V_{k} \cup P_{f}\right)\right]$ not containing $v_{n}$. Faces $f$ and $f^{\prime}$ are not consecutive in the belt of $G_{k}$. Thus, $f$ contains a forbidden vertex $v$. (b, c) If $v$ is forbidden, then (b) $G\left[V \backslash\left(V_{k} \cup P_{f}\right)\right]$ is not connected or (c) there is a singular vertex $w$.

of $w$ in $P_{h_{i}}$ and in $P_{h}$. The cut faces between $h$ and $h_{i}$ do not contain $v$. Hence, by the induction hypothesis, one of them is a locally feasible candidate.

\section{Linear-Time Implementation}

We will maintain a list BELT that represents the cut faces from left to right. For a simpler implementation, BELT contains lists of edges rather than one list of vertices and each cut face $f$ is represented by a belt item which is a pair consisting of

- a list CHAin of $f$ 's incident edges not in $G_{k}$ in clockwise order and

- the rightmost stopper of $P_{f}$ (if any).

We traverse the list BELT using a pointer CANDIDATE.

To decide whether a vertex is a stopper, we maintain two counters. Let $\operatorname{CutFaCES}(v)$ be the number of cut faces and $\operatorname{CuTEDgES}(v)$ the number of cut edges to which $v$ is incident. In order to make the following lemma true also for $v_{n}$, we will count the outer face twice in $\operatorname{CUTFACES}\left(v_{n}\right)$.

Lemma 4. A vertex $v$ in the belt of $G_{k}$ is

- forbidden if and only if $\operatorname{CUTFACES}(v)>\operatorname{CUTEDGES}(v)+1$ and

- singular if and only if $2<\operatorname{CUTFACES}(v)=\operatorname{CUTEDGES}(v)+1$.

Proof. A vertex occurs once for each cut face it is incident to in the belt. Two occurrences of a vertex $v$ in the belt are consecutive if and only if the corresponding cut faces share a cut edge incident to $v$. So, all occurrences of $v$ in the belt are consecutive if and only if $v$ is only incident to one more cut face than to cut edges.

The algorithm canonical0rdering (see Algorithm 2) now works as follows. We start with a copy of $G$ in which each undirected edge $\{v, w\}$ is replaced by the 


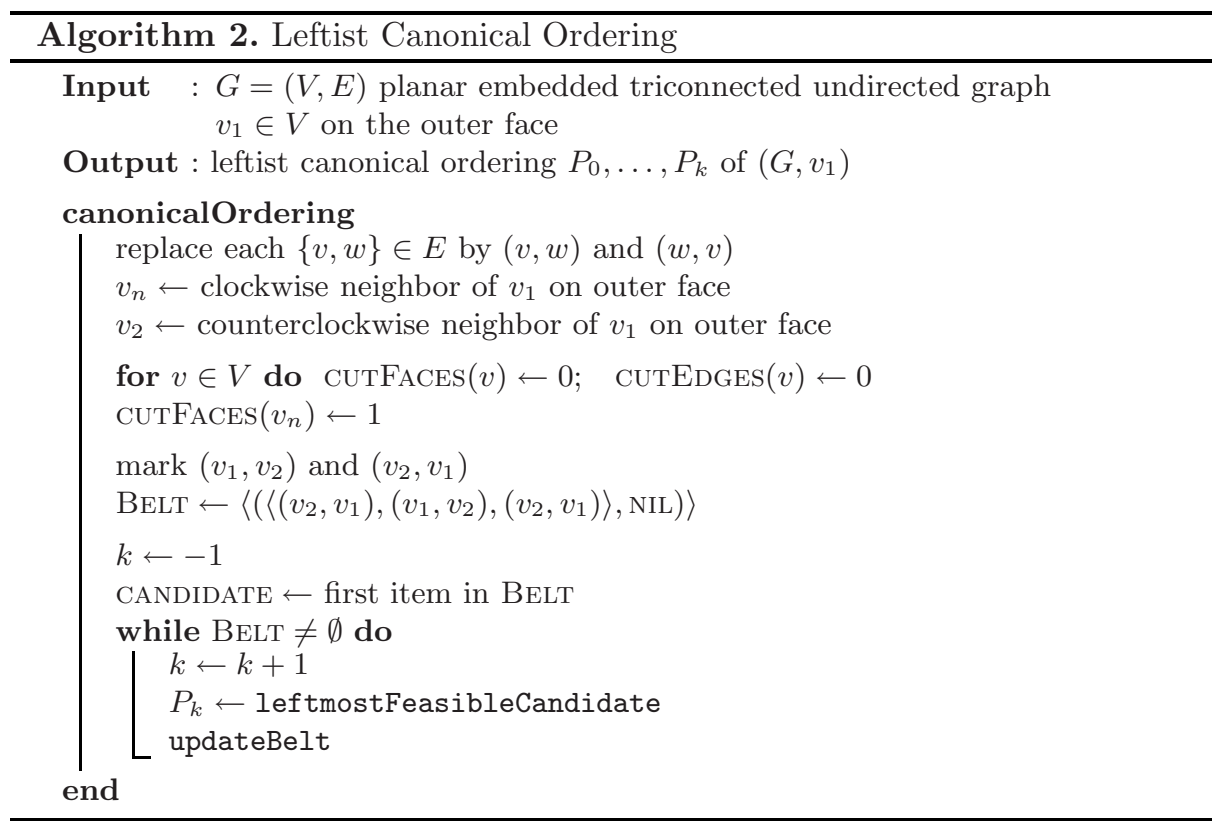

two directed edges $(v, w)$ and $(w, v)$. In the beginning, the belt is initialized by $\left(\left\langle\left(v_{2}, v_{1}\right),\left(v_{1}, v_{2}\right),\left(v_{2}, v_{1}\right)\right\rangle\right.$, NIL). Thus, leftmostFeasibleCandidate (see Algorithm 3 ) chooses $P_{0}=\left\langle v_{1}, v_{2}\right\rangle$ as the first path.

In general, each iteration in Algorithm 2 consists of three steps: (1) We choose the new leftmost locally feasible candidate $P_{k},(2)$ we find the new cut faces incident to $P_{k}$, and (3) we replace $P_{k}$ by its incident cut faces in the belt and update its neighbors (see Fig. 1(c) . In detail:

leftmostFeasibleCandidate. We traverse BELT from the current cut face CANDIDATE to the right doing the following: If CANDIDATE is a candidate face, traverse CANDIDATE. CHAIN from right to left until a stopper is found. If so, store it. If CANDIDATE. CHAIN contains no stopper or it is a singular singleton, it is the next locally feasible candidate. Otherwise, go to the next face. See Algorithm 3 .

beltExtension. To find the new cut faces, we traverse CANDIDATE. CHAIN from left to right. The outer repeat-loop iterates over all vertices incident to two edges of CANDIDATE.CHAIN. Each iteration finds the new cut faces incident to such a vertex and increments the counter CUTEDGES. In the inner repeatloop, we traverse all new edges of a new cut face and store them in the list Chain. Here the counter CUTFACES is incremented. Each list CHAin is finally appended to the list ExTENSION that stores all new belt items incident to CANDIDATE. CHAin. See Algorithm 4.

updateBelt. We replace CANDIDATE (and all its copies if it was a singleton) by the new cut faces found by beltExtension. The last edge of the predecessor 
and the first edge of the successor of CANDIDATE are removed since they are now contained in $G_{k}$. If the predecessor of CANDIDATE was not a candidate face before or it lost its stopper, then we go one step to the left in BELT and set CANDIDATE to its predecessor. See Algorithm 5

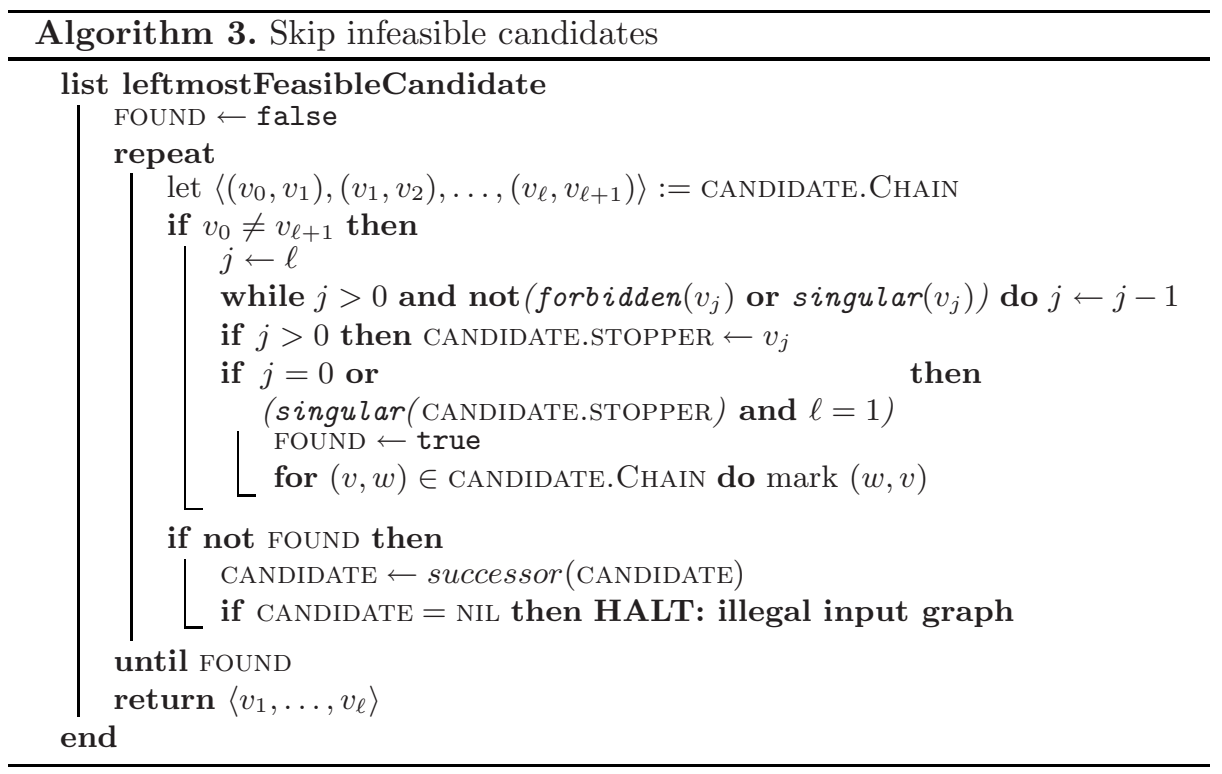

Theorem 2. Algorithm 2 computes the leftist canonical ordering of a triconnected planar graph in linear time.

Proof. Linear running time: In the algorithm beltExtension each edge is touched at most twice. In the algorithm leftmostFeasibleCandidate each candidate is scanned from right to left until the first stopper occurs. All the scanned edges will have been deleted from the list when the candidate will be scanned the next time. In total only $2 m$ edges will be added to BELT.

Correctness: While scanning BELT from left to right, we always choose the leftmost locally feasible candidate: Assume that at step $k+1$ we scan a face $f$ and there are no locally feasible candidates to the left of $f$. The face $f$ is omitted because it is not a candidate or it contains a stopper. None of the two properties changes if no direct neighbor in BELT had been added to $G_{k}$. Hence, as long as $f$ is not locally feasible, no face to the left of $f$ has to be considered. Further, the number of incident cut faces or cut edges of a vertex never decreases. We show that a candidate can only become locally feasible after his rightmost stopper has become singular.

Let $v$ be the rightmost stopper of $P_{f}$ and assume $v$ is forbidden. Let $f_{L}$ be the leftmost and $f_{R}$ be the rightmost cut face containing $v$. We can conclude by the proof of Theorem 1 that all occurrences of $v$ between $f_{L}$ and 

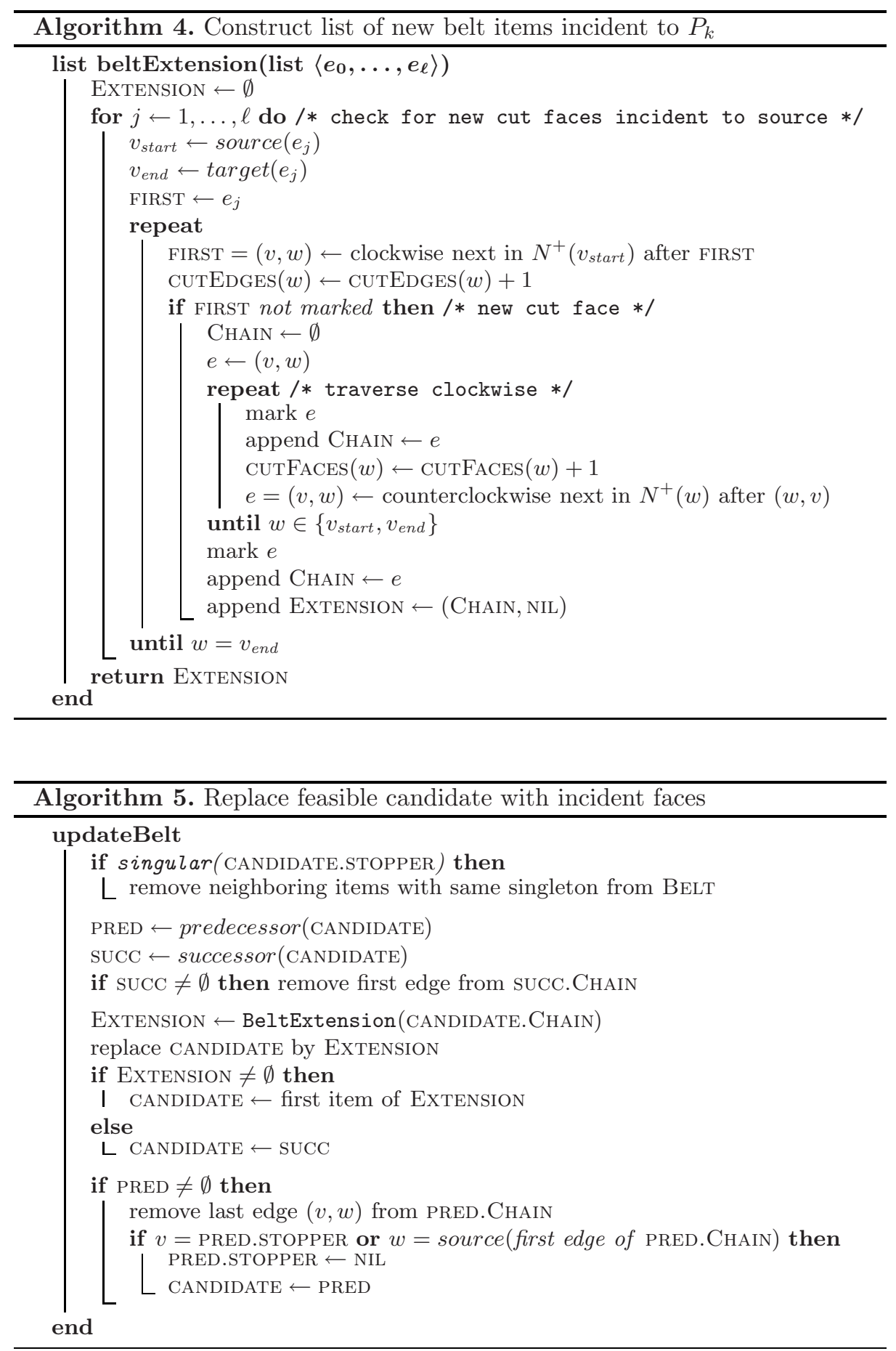
$f$ are consecutive and that Algorithm 2 finds the locally feasible candidates between $f$ and $f_{R}$ in the belt until the belt contains only $v$ between $f$ and $f_{R}$. It follows that all occurrences of $v$ in the belt are consecutive. Let now $v$ be singular. Then, the only two incident cut faces $f=f_{L}$ and $f_{R}$ of $v$ would share a cut edge $\{v, w\}$ that would not have been a cut edge before. Hence, $w$ would have been a stopper of $f$ to the right of $v$.

Note that the algorithm for computing the leftist canonical ordering can also be used to compute the rightist canonical ordering. In that case, we store for each cut face the leftmost stopper and we scan the belt from right to left.

\section{References}

1. Barbay, J., Aleardi, L.C., He, M., Munro, I.: Succinct Representation of Labeled Graphs. In: Tokuyama, T. (ed.) ISAAC 2007. LNCS, vol. 4835, pp. 316-328. Springer, Heidelberg (2007)

2. Barequet, G., Goodrich, M.T., Riley, C.: Drawing Planar Graphs with Large Vertices and Thick Edges. J. of Graph Algorithms and Applications 8(1), 3-20 (2004)

3. Biedl, T.C.: Drawing Planar Partitions I: LL-Drawings and LH-Drawings. In: Proc. 14th Symp. on Computational Geometry, pp. 287-296. ACM Press, New York (1998)

4. Biedl, T.C., Kaufmann, M.: Area-Efficient Static and Incremental Graph Drawings. In: Burkard, R.E., Woeginger, G.J. (eds.) ESA 1997. LNCS, vol. 1284, pp. 37-52. Springer, Heidelberg (1997)

5. Bose, P., Gudmundsson, J., Smid, M.: Constructing Plane Spanners of Bounded Degree and Low Weight. Algorithmica 42(3-4), 249-264 (2005)

6. Brehm, E.: 3-Orientations and Schnyder 3-Tree-Decompositions. Master's thesis, FU Berlin (2000)

7. Chiang, Y.-T., Lin, C.-C., Lu, H.-I.: Orderly Spanning Trees with Applications to Graph Encoding and Graph Drawing. In: Proc. 12th ACM-SIAM Symp. on Discrete Algorithms (SODA 2001), pp. 506-515 (2001)

8. Chrobak, M., Kant, G.: Convex Grid Drawings of 3-Connected Planar Graphs. Int. J. of Computational Geometry and Applications 7(3), 211-223 (1997)

9. Chrobak, M., Nakano, S.-I.: Minimum-Width Grid Drawings of Plane Graphs. Computational Geometry 11(1), 29-54 (1998)

10. Chrobak, M., Payne, T.H.: A Linear-Time Algorithm for Drawing a Planar Graph on a Grid. Information Processing Letters 54(4), 241-246 (1995)

11. Chuang, R.C.-N., Garg, A., He, X., Kao, M.-Y., Lu, H.-I.: Compact Encodings of Planar Graphs via Canonical Orderings and Multiple Parentheses. In: Larsen, K.G., Skyum, S., Winskel, G. (eds.) ICALP 1998. LNCS, vol. 1443, pp. 118-129. Springer, Heidelberg (1998)

12. de Fraysseix, H., Mendez, P.O.: Regular Orientations, Arboricity, and Augmentation. In: Tamassia, R., Tollis, I.G. (eds.) GD 1994. LNCS, vol. 894, pp. 111-118. Springer, Heidelberg (1995)

13. de Fraysseix, H., Pach, J., Pollack, R.: Small Sets Supporting Fáry Embeddings of Planar Graphs. In: Proc. 20th ACM Symp. on the Theory of Computing (STOC 1988), pp. 426-433. ACM Press, New York (1988)

14. de Fraysseix, H., Pach, J., Pollack, R.: How to Draw a Planar Graph on a Grid. Combinatorica 10(1), 41-51 (1990) 
15. Di Battista, G., Tamassia, R., Vismara, L.: Output-Sensitive Reporting of Disjoint Paths. Algorithmica 23(4), 302-340 (1999)

16. Di Giacomo, E., Didimo, W., Liotta, G.: Radial Drawings of Graphs: Geometric Constraints and Trade-offs. J. of Discrete Algorithms 6(1), 109-124 (2008)

17. Di Giacomo, E., Didimo, W., Liotta, G., Wismath, S.K.: Curve-Constrained Drawings of Planar Graphs. Computational Geometry 30(2), 1-23 (2005)

18. Dujmović, V., Suderman, M., Wood, D.R.: Really Straight Graph Drawings. In: GD 2004 [21], pp. 122-132

19. Erten, C., Kobourov, S.G.: Simultaneous Embedding of Planar Graphs with Few Bends. In: GD 2004 [21], pp. 195-205

20. Fößmeier, U., Kant, G., Kaufmann, M.: 2-Visibility Drawings of Planar Graphs. In: North, S.C. (ed.) GD 1996. LNCS, vol. 1190, pp. 155-168. Springer, Heidelberg (1997)

21. Pach, J. (ed.): GD 2004. LNCS, vol. 3383. Springer, Heidelberg (2005)

22. Goodrich, M.T., Wagner, C.G.: A Framework for Drawing Planar Graphs with Curves and Polylines. J. of Algorithms 37(2), 399-421 (2000)

23. Gutwenger, C., Mutzel, P.: Planar Polyline Drawings with Good Angular Resolution. In: Whitesides, S.H. (ed.) GD 1998. LNCS, vol. 1547, pp. 167-182. Springer, Heidelberg (1999)

24. Harel, D., Sardas, M.: An Algorithm for Straight-Line Drawing of Planar Graphs. Algorithmica 20, 119-135 (1998)

25. He, X.: On Floor-Plan of Plane Graphs. SIAM J. on Computing 28(6), 2150-2167 (1999)

26. He, X., Kao, M.-Y., Lu, H.-I.: Linear-Time Succinct Encodings of Planar Graphs via Canonical Orderings. SIAM J. on Discrete Mathematics 12(3), 317-325 (1999)

27. Kant, G.: Drawing Planar Graphs using the lmc-Ordering. Technical Report RUUCS-92-33, Dep. of Information and Computing Sciences, Utrecht University (1992)

28. Kant, G.: Drawing Planar Graphs Using the Canonical Ordering. Algorithmica 16(4), 4-32 (1996)

29. Kant, G.: A More Compact Visibility Representation. Int. J. of Computational Geometry and Applications 7(3), 197-210 (1997)

30. Kant, G., He, X.: Regular Edge Labeling of 4-Connected Plane Graphs and its Applications in Graph Drawing Problems. Theoretical Computer Science 172(12), 175-193 (1997)

31. Miura, K., Azuma, M., Nishizeki, T.: Canonical Decomposition, Realizer, Schnyder Labeling and Orderly Spanning Trees of Plane Graphs. Int. J. of Foundations of Computer Science 16(1), 117-141 (2005)

32. Nakano, S.-I.: Planar Drawings of Plane Graphs. IEICE Transactions on Information and Systems E83-D(3), 384-391 (2000)

33. Schnyder, W.: Embedding Planar Graphs on the Grid. In: Proc. 1st ACM-SIAM Symp. on Discrete Algorithms (SODA 1990), pp. 138-148 (1990)

34. Wada, K., Chen, W.: Linear Algorithms for a $k$-Partition Problem of Planar Graphs. In: Hromkovič, J., Sýkora, O. (eds.) WG 1998. LNCS, vol. 1517, pp. 324 336. Springer, Heidelberg (1998) 